\title{
O aprimoramento para enfermeiras obstétricas: protocolo de uma scoping review
}

\author{
Improvement for obstetric nurses: a scoping review protocol \\ Mejora para las enfermeras obstétricas: un protocolo de revisión del alcance
}

Recebido: 09/09/2021 | Revisado: 17/09/2021 | Aceito: 21/09/2021 | Publicado: 23/09/2021

Thalita Rocha Oliveira

ORCID: https://orcid.org/0000-0003-3316-4880 Universidade Federal Fluminense, Brasil

E-mail: 15oliveira.thalita@gmail.com

Valdecyr Herdy Alves

ORCID: https://orcid.org/0000-0001-8671-5063 Universidade Federal Fluminense, Brasil

E-mail: herdyalves@yahoo.com.br

Bianca Dargam Gomes Vieira

ORCID: https://orcid.org/0000-0002-0734-3685 Universidade Federal Fluminense, Brasil

E-mail: biadargam@gmail.com

Audrey Vidal Pereira

ORCID: https://orcid.org/0000-0002-6570-9016 Universidade Federal Fluminense, Brasil E-mail: avpereira@id.uff.br

Juliana Vidal Vieira Guerra

ORCID: https://orcid.org/0000-0002-4509-1343

Universidade Federal Fluminense, Brasil

E-mail: julianaguerra.personaldiet@gmail.com

Diego Pereira Rodrigues

ORCID: https://orcid.org/0000-0001-8383-7663

Universidade Federal do Pará, Brasil

E-mail: diego.pereira.rodrigues@gmail.com

Paolla Amorim Malheiros Dulfe

ORCID: https://orcid.org/0000-0003-1653-4640

Universidade Federal Fluminense, Brasil

E-mail: paolla_amorim@yahoo.com.br

\begin{abstract}
Resumo
Objetivo: Mapear a extensão e o alcance das evidências sobre aprimoramento em saúde materno infantil e seu impacto na prática de enfermeira(o)s obstétrica(o)s. Metodologia: Protocolo de scoping review segundo o Joanna Briggs Institute Review's Manual. Incluídas pesquisas sobre o aprimoramento em saúde materno-infantil e os impactos nas práticas (conceito) da(o)s enfermeira(o)s obstétrica(o)s (participante) nos serviços de saúde (contexto). Serão realizadas buscas nas bases de dados: LILACS, BDENF, MEDLINE, EMBASE e SCOPUS, no período entre outubro de 2021 a janeiro de 2022, nos idiomas português, inglês e espanhol. Para a etapa de seleção dos estudos, o time de revisão realizará de forma independente a seleção utilizando-se de ferramenta de extração de dados. Resultados: Os dados serão apresentados de modo tabular, diagramática e descritiva a partir das evidências encontradas e reunidos em categorias para análise na modalidade temática. Conclusão: Espera-se contribuir para processo reflexivo sobre a qualificação e valorização do cuidado da enfermeira(o) obstétrica(o).
\end{abstract}

Palavras-chave: Enfermagem obstétrica; Educação em enfermagem obstétrica; Formação profissional; Tocologia; Assistência perinatal; Serviços de saúde.

\begin{abstract}
Objective: To map the extent and reach of evidence on improvement in maternal and child health and its impact on the practice of obstetric nurses. Method: Scoping review protocol according to the Joanna Briggs Institute Review's Manual. Included researches on the improvement of maternal and child health and the impacts on the practices (concept) of obstetric nurses (participant) in health services (context). Searches will be carried out in the following databases: LILACS, BDENF, MEDLINE, EMBASE and SCOPUS, from October to January 2022, in Portuguese, English and Spanish. For the study selection stage, the review team will independently carry out the selection using a data extraction tool. Results: The data will be presented in a tabular, diagrammatic and descriptive way from the evidence found and gathered into categories for analysis in the thematic modality. Conclusion: It is expected to contribute to the reflective process on the qualification and valorization of the obstetric nurse's care.
\end{abstract}

Keywords: Obstetric nursing; Obstetric nursing education; Professional training; Tocology; Perinatal care; Health services. 


\begin{abstract}
Resumen
Objetivo: Mapear la extension y el alcance de las evidencias sobre mejora en salud materno infantil y su impacto em la practica de enfermera(o)s obstétrica(o)s. Metodología: Protocolo de revisión del alcance según el Manual de revisión del Instituto Joanna Briggs. Se incluyeron investigaciones sobre el mejoramiento de la salud materno infantil y los impactos en las prácticas (concepto) de las enfermeras obstétricas (participante) en los servicios de salud (contexto). Se realizarán búsquedas en las siguientes bases de datos: LILACS, BDENF, MEDLINE, EMBASE y SCOPUS, de octubre de 2021 a enero de 2022, en portugués, inglés y español. Para la etapa de selección del estudio, el equipo de revisión llevará a cabo la selección de forma independiente utilizando una herramienta de extracción de datos. Resultados: Los datos se presentarán de forma tabular, esquemática y descriptiva a partir de la evidencia encontrada y reunidos en categorías para su análisis en la modalidad temática. Conclusión: Se espera contribuir al proceso de reflexión sobre la calificación y valorización del cuidado de la enfermera obstétrica.
\end{abstract}

Palabras clave: Enfermería obstétrica; Educación en enfermería obstétrica; Formación professional; Tocología; Atención perinatal; Servicios de salud.

\title{
1. Introdução
}

A enfermagem vivencia momento de reconhecimento e valorização profissional, estando na linha de frente de muitos cenários de cuidado em saúde, em especial no protagonismo no desempenho dos cuidados em saúde durante o curso da pandemia de covid-19 (Sousa et al., 2020). Mendes et al. (2020) cita que em 2018 através de colaboração entre Organização Mundial de Saúde (OMS) e o Conselho Internacional de Enfermeiros (ICN) é lançada a Campanha Nursing Now, a qual defende o fortalecimento e o investimento na profissão assim como a qualificação da educação de enfermeiros, por meio da melhoria do ensino e do desenvolvimento profissional. Adicionalmente, em 2020, além de comemorar-se o bicentenário de Florence Nightingale, declarou-se o "Ano dos Profissionais de Enfermagem e Obstetrícia” num reconhecimento da OMS pelo trabalho das enfermeiras e parteiras em todo o mundo, o que impulsionou o aumento de investimento nesta força de trabalho, melhoria das condições para atuação, educação e desenvolvimento profissional (Reynolds, 2020). Assim, na atualidade, sobressalta-se que sem a valorização e qualificação das enfermeiras e obstetrizes, ou seja, uma educação em obstetrícia de qualidade, não há base estruturada para o pleno cuidado à saúde e cumprimento da agenda de desenvolvimento sustentável.

A qualificação das profissionais que atuam no campo obstétrico, de forma integrada com o sistema de saúde e em equipes multiprofissionais, é uma estratégia que possibilita a melhoria da qualidade do atendimento que traz como resultados não só a redução em até $80 \%$ das mortes maternas e neonatais, mas também outros resultados positivos como redução da morbidade, menor uso de intervenções durante o trabalho de parto e parto, maior satisfação com a vivência da gestação e parto e melhores resultados no sucesso do aleitamento materno (Who, 2019).

No que tange ao modelo de cuidado obstétrico, liderados pelas midwifes, definido por Who (2020) como aquela profissional qualificada para o cuidado a mulheres durante a gravidez, parto e pós-parto e recém nascidos nas primeiras semanas de vida, considerando a fisiologia do processo, as quais possuem ampla atuação há décadas. O que favorece a redução das intervenções no parto, melhora dos resultados psicossociais e da morbidade materna e neonatal, representando uma assistência obstétrica de qualidade, fundamental para alcançar as prioridades nacionais e mundiais e atingir a cobertura universal da saúde. Garantindo deste modo os direitos das mulheres e de seus recém-nascidos (Sandall et al., 2016). Assim, países que apresentam alto Índice de Desenvolvimento Humano (IDH) e contam com a atuação destas profissionais registraram em 2012 taxas de cesariana em torno de 27\% e taxa de mortalidade materna em 16,2 por cem mil nascidos vivos em 2010 (Ye et al., 2016).

No Brasil, há alguns anos apresenta-se dificuldades de enfrentamento das taxas de cesarianas. Dados apontam que, em 2016, 55,5\% dos partos ocorreram via cesariana (Brasil, 2017), o que traz também evidentes desdobramentos na razão da mortalidade materna que, em 2018, registrou 59,1 óbitos para cada cem mil nascidos vivos, meta distante daquela definida pelos Objetivos do Desenvolvimento Sustentável para 2030 (Brasil, 2020). Este é um reflexo da qualidade da assistência oferecida e do tradicional modelo de atenção obstétrico brasileiro configurado por ações de cunho individualizado, 
biologicista, tecnocrático, com baixa adesão às boas práticas de atenção ao parto e nascimento, as quais valorizam o cuidado ao corpo feminino e sua fisiologia (Paula et al., 2020).

Este desenho retrata um desafio para órgãos governamentais responsáveis pela elaboração de políticas públicas na área da atenção à Saúde da Mulher há algumas décadas, considerando os princípios do SUS e as mais recentes evidências científicas sobre ações profissionais e Institucionais a serem implementadas a partir de normativas. Uma das estratégias adotadas pelo Ministério da Saúde (MS) foi a implementação da Rede Cegonha (Brasil, 2011) e do Projeto Aprimoramento e Inovação no Cuidado e Ensino em Obstetrícia e Neonatologia (ApiceOn) (Brasil, 2017) na tentativa de reconfiguração deste modelo através da formação e qualificação profissional das enfermeiras obstétricas, seja através do financiamento de Cursos de Especialização e Programas de Residência em Enfermagem Obstétrica (Oliveira et al., 2016) como também pelos Cursos de Aprimoramento em Enfermagem Obstétrica executados por diversas Instituições de Ensino Superior (Carlos et al., 2019; Souza et al., 2019) por compreender que estas profissionais são importantes no processo assistencial, fortalecendo mudanças e readequação nas práticas obstétricas (Vargens et al., 2017; Guida et al., 2017).

Nesse sentido, considerando a relevância e atualidade da temática do aprimoramento de enfermeira(o)s obstétrica(o)s, a necessidade de investimentos financeiros no desenvolvimento da enfermagem e a capacitação profissional de enfermeira(o)s obstétrica(o)s, com finalidade de impacto positivo dos indicadores de saúde perinatais por meio do cuidado qualificado. Deste modo, este estudo se objetiva a mapear a extensão e o alcance das evidências sobre aprimoramento em saúde materno infantil e seu impacto na prática de enfermeira(o)s obstétrica(o)s. Assim, trazer a luz o conhecimento advindo destas produções para possibilitar identificar os avanços na qualidade, seus impactos e as ações em saúde desempenhadas pelas enfermeiras(o)s obstétrica(o)s em sua prática a partir do aperfeiçoamento profissional. Entendendo que estas constituem-se base, estrutura, fundamento, um agente de movimento, de diferentes sistemas de saúde e para o desenvolvimento e alcance de metas e objetivos de políticas públicas definidas internacionalmente. Torna-se evidente frisar que o resultado do exercício profissional da enfermeira(o) obstétrica(o) qualificada contribui para a expansão, fortalecimento e valorização da categoria.

\section{Metodologia}

Este protocolo de scoping review, será conduzido seguindo as recomendações de metodologia do JBI para scoping review (Peters et al., 2020) e foi registrado no OSF (Research and data management software for open Science) sob o DOI 10.17605/OSF.IO/U9JF3. Consiste em uma etapa preliminar de uma revisão de escopo, cujo objetivo é mapear as evidências existentes sobre determinado assunto sem uma análise da qualidade metodológica dos estudos incluídos, pois a intenção não é identificar a melhor evidência e sim, reuni-las apresentando os principais conceitos, teorias e lacunas do conhecimento, bem como analisar como determinada pesquisa é realizada a partir de um tópico ou campo (Munn et al., 2018; Colquhoun et al., 2014).

\section{Pergunta de revisão}

Como o aprimoramento em saúde materno-infantil impacta na prática das enfermeiras(o)s obstétrica(o)s?

\section{Critérios de Inclusão}

\section{Participantes}

Serão considerados estudos cujos participantes sejam enfermeira(o)s obstétrica(o)s, profissionais habilitados de acordo com regulamentação de cada país para exercer cuidados especializados a mulheres e seus recém nascidos, que realizaram algum curso de aprimoramento profissional. Serão desconsiderados estudos, cujos participantes sejam enfermeira(o)s sem a especialização na área obstétrica ou outros membros da equipe de saúde. 


\section{Conceito}

Serão considerados estudos que versem sobre o aprimoramento profissional de enfermeira(o)s obstétrica(o)s e as práticas destes profissionais.

\section{Contexto}

Serão considerados estudos que versem sobre o contexto nacional dos serviços de saúde materno-infantil públicos e privados, e a nível internacional.

\section{Tipos de fontes de Informação}

Serão considerados estudos do tipo investigações primárias e secundárias, assim como estudos quantitativos observacionais e experimentais. Para pesquisas qualitativas incluiremos desenhos metodológicos variados e relatos de experiência oriundos de dissertações, teses de acesso livre e artigos com textos disponíveis na íntegra e com livre acesso, nos idiomas português, inglês e espanhol publicados de 01 de maio de 2011 a 01 setembro de 2021. Não serão considerados os estudos que não contemplem a temática de aprimoramento em saúde materno-infantil e que tenham como participantes outros profissionais de saúde diferentes de enfermeira(o)s obstétrica(o)s, materiais não disponíveis eletronicamente em sua versão completa no período de realização das buscas e seleção e publicações como cartas ao editor, opinião de especialistas e resenhas.

\section{Estratégia de busca}

A estratégia de busca se limitará a estudos publicados e não-publicados. Foi desenvolvida busca piloto em abril de 2020, nas bases de dados: CINAHL (Cumulative Index to Nursing and Allied Health Literature) e no Banco de Teses e Dissertações da CAPES (Coordenação de Aperfeiçoamento de Pessoal de Nível Superior) a fim de avaliar o alcance temático e a identificação de termos de indexação e palavras-chave (Tabela 1). 
Tabela 1- Pesquisa conduzida em abril de 2021: estratégia similar será utilizada em outras bases de dados.

\begin{tabular}{|c|c|c|c|c|c|c|c|}
\hline Base de dados & Cinahal* & Achados (n) & Achados (\%) & BDTD $^{* *}$ & Achados (n) & Achados (\%) & Total (n) \\
\hline Pesquisa 1 & $\begin{array}{l}\text { "Professional } \\
\text { training" OR } \\
\text { "Health human } \\
\text { resource } \\
\text { training" }\end{array}$ & 2.325 & 94,17 & $\begin{array}{l}\text { "Capacitação } \\
\text { profissional" OR } \\
\text { "Capacitação de } \\
\text { recursos } \\
\text { humanos em } \\
\text { saúde" }\end{array}$ & 144 & 5,83 & 2469 \\
\hline Pesquisa 2 & $\begin{array}{c}\text { "Nurse } \\
\text { midwives" OR } \\
\text { "obstetric } \\
\text { nursing" }\end{array}$ & 6.831 & 97,29 & $\begin{array}{l}\text { "Enfermeiras } \\
\text { obstétricas" OR } \\
\text { "Enfermagem } \\
\text { obstétrica" }\end{array}$ & 190 & 2,71 & 7021 \\
\hline Pesquisa 3 & $\begin{array}{l}\text { "Maternal- } \\
\text { child health } \\
\text { service" OR } \\
\text { "Hospitals, } \\
\text { maternity" }\end{array}$ & 271 & 42,74 & $\begin{array}{l}\text { "Serviços de } \\
\text { saúde materno- } \\
\text { infantil" OR } \\
\text { "Maternidades" }\end{array}$ & 363 & 57,26 & 634 \\
\hline Pesquisa 4 & $\begin{array}{c}\text { "Perinatal } \\
\text { care" OR } \\
\text { "Midwifery" }\end{array}$ & 7527 & 99,97 & $\begin{array}{l}\text { "Assistência } \\
\text { perinatal" OR } \\
\text { "Tocologia" }\end{array}$ & 2 & 0,03 & 7529 \\
\hline Pesquisa 5 & $\begin{array}{c}1 \text { OR } 2 \text { OR } 3 \\
\text { OR } 4\end{array}$ & 15242 & 100 & $\underset{4}{1 \text { OR } 2 \text { OR } 3 \text { OR }}$ & 0 & 0 & 15242 \\
\hline Pesquisa 6 & 1 AND 2 & 110 & 98,21 & 1 AND 2 & 2 & 1,79 & 112 \\
\hline Pesquisa 7 & 1 AND 3 & 3 & 2,13 & 1 AND 3 & 138 & 97,87 & 141 \\
\hline Pesquisa 8 & 1 AND 4 & 147 & 65,62 & 1 AND 4 & 77 & 34,38 & 224 \\
\hline Pesquisa 9 & $\begin{array}{c}1 \text { AND } 2 \text { AND } \\
3 \text { AND } 4\end{array}$ & 1 & 100 & $\begin{array}{c}1 \text { AND } 2 \text { AND } 3 \\
\text { AND } 4\end{array}$ & 0 & 0 & 1 \\
\hline
\end{tabular}

As pesquisas realizadas no mês de abril de 2021 consideraram os idiomas inglês, português e espanhol.

* Cinahal- Cumulative Index to Nursing and Allied Health Literature

**BDTD- Banco de Teses e Dissertações

Fonte: Autores (2021)

Podemos observar na tabela acima que inicialmente realizou-se a busca isolada dos descritores definidos nesta pesquisa a partir da mnemônica PCC (participantes, conceito, contexto), o que gerou achados para cada base de dados. Em seguida, prosseguiu-se a pesquisa com a associação de todos os descritores, que possibilita melhor levantamento das evidências sobre a pergunta de revisão nesta fase de busca piloto.

Em seguida, as palavras chaves e descritores utilizados nos artigos relevantes identificados, foram utilizadas para elaboração de uma estratégia de busca completa. Assim, elegeram-se os seguintes descritores Enfermeira obstétrica, Enfermagem obstétrica, Capacitação profissional, Capacitação de recursos humanos em saúde, Desenvolvimento de pessoal, Educação continuada em Enfermagem, Tocologia, Parto, Assistência Perinatal, Serviços de Saúde Materno Infantil, Sala de Parto, Maternidades, Hospitais. A estratégia de busca, incluindo as palavras-chaves e termos indexadores identificados serão adaptados para cada base de dados. A lista de referência de todas as fontes de informação incluída será considerada para seleção de possíveis estudos adicionais. Serão considerados os estudos em língua inglesa, espanhola e portuguesa.

\section{Seleção das fontes de evidência}

Posterior a etapa da busca ampliada, as publicações identificadas serão selecionadas, listadas e seu texto completo transferido em pdf para gerenciador de referências Mendeley, com remoção das duplicatas. 
Dois revisores independentes realizarão a leitura atentiva dos títulos e resumos a partir dos critérios de inclusão definidos nesta revisão. Os textos completos após selecionados serão analisados detalhadamente pelo time de revisores. Aqueles que não se enquadrarem nos critérios de inclusão delimitados serão excluídos com registro e explicitação do motivo pelo qual não fizeram parte do escopo de análise. Caso haja divergências entre os dois revisores, um encontro virtual para diálogo se fará necessário para um consenso ou a consulta a um terceiro revisor para confirmar a elegibilidade do estudo.

As etapas de inclusão e exclusão dos estudos, assim como os resultados das pesquisas serão descritos integralmente na revisão de escopo final e apresentados segundo modelo de diagrama de fluxo de Itens de Relatório para Revisões Sistemáticas e Meta-análises para revisão de escopo (PRISMA-ScR) (Trico et al., 2018).

\section{Extração dos dados}

A partir dos textos completos selecionados, serão extraídos os dados por dois revisores independentes com auxílio de instrumento elaborado pelos autores (Figura 1) para registro das informações, a partir de um modelo de instrumento para detalhes da fonte, características e extração de resultados (Peters et al., 2020).

Figura 1: Instrumento de extração de dados e características do estudo.

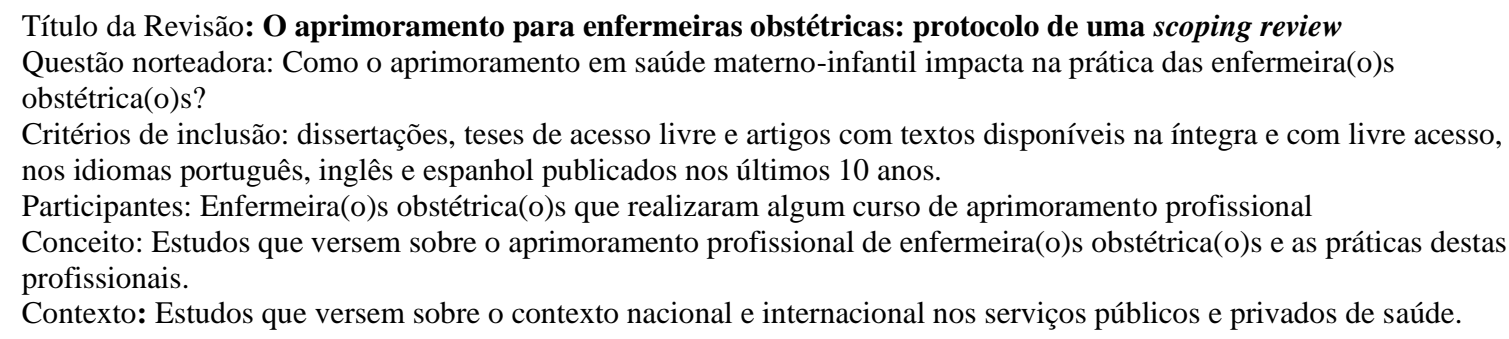

\begin{tabular}{|c|c|}
\hline Dados Estudo & Descrição informações \\
\hline \multicolumn{2}{|l|}{ Autores } \\
\hline \multicolumn{2}{|l|}{ Ano de publicação } \\
\hline \multicolumn{2}{|l|}{ Título } \\
\hline \multicolumn{2}{|l|}{ Tipo de publicação: Monografias, dissertações, teses, artigos } \\
\hline \multicolumn{2}{|l|}{ Local do estudo: País, Estado, Cidade } \\
\hline \multicolumn{2}{|l|}{ Objetivo e propósito do estudo } \\
\hline \multicolumn{2}{|l|}{ Metodologia } \\
\hline $\begin{array}{cl}\text { População } \\
\circ & \text { Participantes alvo da pesquisa } \\
\circ & \text { Número de integrantes } \\
\circ & \text { Critérios de inclusão e exclusão } \\
\end{array}$ & \\
\hline $\begin{array}{ll}\text { Curso de } & \text { aprimoramento } \\
\circ & \text { Descrição do objetivo } \\
\circ & \text { Temática e metodologia do curso de } \\
& \text { aprimoramento realizado } \\
\end{array}$ & \\
\hline $\begin{array}{c}\text { Resultados } \\
\quad \begin{array}{l}\text { Descrição dos principais conceitos e achados } \\
\text { relacionados à população, conceito e contexto }\end{array} \\
\end{array}$ & \\
\hline $\begin{array}{l}\text { Conclusões } \\
\quad \text { Descrição de aspectos positivos, negativos, } \\
\text { especificidades relacionadas à população, conceito } \\
\text { e contexto }\end{array}$ & \\
\hline
\end{tabular}


Assim serão extraídos dados que irão fornecer o detalhamento das pesquisas relativos à população, cenário, métodos e resultados que contemplem a questão norteadora e objetivo desta revisão de escopo. Um teste piloto para familiarização do instrumento pelos revisores será aplicado. Após, no curso da análise, caso haja necessidade de modificação do instrumento de extração com acréscimo de informações pertinentes, esta será realizada entendendo que se constitui em uma ferramenta preliminar, sendo declaradas na revisão de escopo. Caso sejam necessárias informacionais adicionais, os revisores entrarão em contato com a autoria do material analisado para maior detalhamento.

\section{Análise e apresentação dos resultados}

Os dados levantados mediante à questão desta revisão de escopo que se relacionam ao período, tipo de publicação, local de estudo, cenário, população e metodologia serão apresentados por meio de tabela, gráficos e figuras representativas. Já a coletânea de evidências disponíveis sobre os conceitos, principais características, fatores relacionados, aspectos facilitadores e dificultadores ao tema que está sendo pesquisado, os desdobramentos do aprimoramento em saúde materno infantil para a prática da(o) enfermeira(o)s obstétrica(o)s nos serviços de saúde materno infantil, passarão por descrição e narração das informações por meio de análise de conteúdo na modalidade temática (Bardin, 2011), contribuindo assim para apresentar as evidências sobre a qualificação profissional no exercício do cuidado da enfermeira(o) obstétrica(o) no campo da saúde materna e perinatal.

\section{Conclusão}

Entendendo que os cursos de aprimoramento em saúde materno infantil se constituem canais para capacitação profissional a fim de atualizar conhecimentos científicos e qualificar habilidades técnicas e relacionais para o exercício da profissão, a partir de um cuidado científico, individualizado, humanizado e social, destaca-se o mérito na divulgação de evidências disponíveis sobre esta temática por meio da realização desta scoping review.

Desta forma, ao apresentar e analisar o conhecimento produzido sobre o impacto na prática das enfermeiras(o)s obstétrica(o)s nos serviços de saúde materno infantil a partir da realização de cursos de aprimoramento profissional, espera-se fomentar ferramentas para o processo de atenção e gestão do cuidado.

Ademais, ampliar o conhecimento destes resultados à comunidade acadêmica, contribui para a formulação, continuidade e desenvolvimento de novos cursos de aprimoramento profissional pelas Instituições de Ensino, favorecendo uma capacitação em conhecimentos técnico-científicos que atendam a demanda das necessidades das enfermeiras, dos serviços e da sociedade. Além, de fornecer dados para reflexão no que tange a formulação e redirecionamento de políticas públicas que englobem a assistência à saúde materno infantil considerando seus direitos e garantias, bem como campanhas de valorização da enfermagem, que em seu exercício profissional é responsável pela oferta de cuidado seguro, benéfico e de qualidade.

Por meio da revisão de escopo, etapa subsequente a este protocolo, almeja-se que seja um ponto de partida para futuras pesquisas a partir das evidências apresentadas e com isso, possibilitar avaliar in loco o impacto do aprimoramento profissional da enfermeira obstétrica nos serviços de saúde materno infantil por meio da qualificação do seu cuidado e assim, ratificar as recomendações internacionais para o desenvolvimento de políticas e ações neste âmbito.

\section{Referências}

Bardin L. (2011). Análise de conteúdo. Edições 70.

Brasil. Ministério da Saúde. Boletim Epidemiológico [Internet]. (2020). Secretaria de Vigilância em Saúde, 20(51),1-47. https://portaldeboaspraticas.iff.fiocruz.br/biblioteca/boletim-epidemiologico-no-20-maio-2020/

Brasil. Ministério da Saúde. Manual Prático para Implementação da Rede Cegonha [Internet]. (2011). Secretaria de Políticas de Saúde. http://www.saude.pi.gov.br/ckeditor_assets/attachments/138/Documentos_rede_cegonha.pdf 
Brasil. Ministério da Saúde. ApiceOn - Aprimoramento e Inovação no Cuidado e Ensino em Obstétricia e Neonatologia [Internet]. (2017). Secretaria de Atenção à Saúde. Departamento de Ações Programáticas e Estratégicas.

https://portaldeboaspraticas.iff.fiocruz.br/apice/o-projeto/

Carlos, G. A. V., Matozinhos, F. P., Carmo, J. M. A do., Manzo, B. F., Duarte, E. D. \& Souza, K. V. (2019). Perfil dos participantes de um curso de aprimoramento em enfermagem obstétrica. Rev Mineira Enferm, 23(e-1153), 1-7. http://doi: 10.5935/1415-2762.20190006

Colquhoun, H. L., Levac, D., O’Brien, K. K., Strauss, S., Tricco, A. C., Perrier, L., Kastner, M. \& Moher, D. (2014). Scoping review: time for clarity in definition, methodos and reporting. Journal of Clinical Epidemiology, 67(12), 1291-1294. https://doi.org/10.1016/j.jclinepi.2014.03.013

Laboissière, P. (2017, 10 de março). Número de cesarianas cai pela primeira vez no Brasil. Empresa Brasil de Comunicação - Agência Brasil. https://agenciabrasil.ebc.com.br/geral/noticia/2017-03/numero-de-cesarianas-cai-pela-primeira-vez-no-brasil

Mendes, I. A. C., Ventura, C. A. A., Silva, M. C. N da., Lunardi, V. L., Silva, I. R. \& Santos, S. S. dos. (2020). Nursing now and Always: evidence for the implementation of the Nursing Now campaign. Rev Lat Am Enfermagem, 28(e3388), 1-11. http://doi: 10.1590/1518-8345.4553-3388.

Munn, Z., Peters, M. D. J., Stern, C., Tufanaru, C., McArthur, A. \& Aromataris, E. (2018). Systematic Review or Scoping Review? Guidance for authors when choosing between a systematic or a scoping review approach. BMC Medical Research Metodology, 18(1), 143. https://doi.org/10.1186/s12874-018

Oliveira, F. A. M., Leal, G. C. G., Wolf, L. D. G., Rabelo, M. \& Poliquesi, C. B. Reflexões acerca da atuação do enfermeiro na Rede de Cegonha. (2016). Rev Enferm UFPE, 10(supl 2), 867-74. http://doi: 10.5205/reuol.6884-59404-2-SM-1.1002sup201622

Paula, E de., Alves, V. H., Rodrigues, D. P., Felicio, F. de C., Araújo, R. C. B. de, Chamilco, R, A, da S. I \& Almeida, V. L. M. (2020). Violência Obstétrica e o atual modelo obstétrico, na percepção dos gestores em saúde. Texto Contexto Enferm, 29(e2019248). http:// doi: org/10.1590/1980-265X-TCE-2019-0248

Peters, M. D. J., Godfrey, C., McInerney, P., Munn, Z., Tricco, A. C., \& Khalil, H. (2020). Chapter 11: Scoping Reviews. In: Aromataris, E., Munn, Z. (Eds.), JBI Manual for Evidence Synthesis [Internet]. https://synthesismanual.jbi.global

Reynolds, N. R. (2020). The year of the nurse and midwife 2020: activating the potential and power of nursing. Rev Lat A. Enfermagem, 28(e3279),1-3. http://doi: 10.1590/1518-8345.0000-3279.

Sandall, J., Soltani, H., Gates, S., Shennan, A. \& Devane, D. (2016). Midwife-led continuity models versus other models of care for childbearing women. Cochrane Database of Systematic Reviews. Issue 4. http://doi: 10.1002/14651858.CD004667.

Sousa, A. R. de, Santos, G. L. A., Silva, R. S. da \& Carvalho, E. S. de S. (2020). Reflexões sobre o processo de enfermagem no trabalho de enfermeiras frente à pandemia da COVID-19. Enferm Foco, 11(1), 62-67. http://dx.doi.org/10.21675/2357-707X.2020.v11.n1.ESP.3501

Souza, K. V. de (orgs). (2019). Caderno do Curso de Aprimoramento em Enfermagem Obstétrica com foco na atenção ao parto e nascimento: qualificação dos processos de cuidado e de gestão (CAEO/PN/APICEON). Escola de Enfermagem da Universidade Federal de Minas Gerais.

Tricco, A. C., Lillie, E., Zarin, W., O’Brien, K. K., Colquhoun, H. \& Levac, D. (2018). PRISMA Extension for Scoping Reviews (PRISMA-ScR): Checklist and Explanation. Ann Intern Med, 169(7), 467-473. http://doi10.7326/M18-0850

Vargens, O. M. da C., Silva, A. C. V. da \& Progianti, J. M. (2017). Contribuição de Enfermeiras Obstétricas para consolidação do parto humanizado em maternidades no Rio de Janeiro-Brasil. Esc Anna Nery Rev de Enferm, 21(1), 1-8. 2017. http://doi10.5935/1414-8145.20170015.

World Health Organization (2019). Strengthening quality midwifery education for Universal Health Coverage 2030: framework for action [Internet]. https://apps.who.int/iris/bitstream/handle/10665/324738/9789241515849eng.pdf?ua=

World Health Organization (2020). The case for midwifery. 2020 [Internet].

https://www.who.int/maternal_child_adolescent/topics/qualityofcare/midwifery/case-for-midwifery/en/

Ye, J., Zhang, J., Mikolajczyk, R., Torloni, M. R., Gülmezoglu, A. M. \& Betran, A. P. (2026). Association between rates of caesarean section and maternal and neonatal mortality in the 21st century: a worldwide population-based ecological study with longitudinal data. BJOG: An International Journal of Obstetrics and Ginaecology, 123(5), 745-53. http://doi10.1111/1471-0528.13592. 